*Supplementary Information

\title{
Integration of Nitrogen-Doped Graphene Oxide
}

\section{Dots with Au Nanoparticles for Enhanced}

\section{Electrocatalytic Hydrogen Evolution}

Naveen Kumar Reddy Bogireddy, ${ }^{[a]}$ Abdel Ghafour El Hachimi, ${ }^{[b]}$ Jesús Muñiz, ${ }^{[b]}$ Ana

Laura Elías, ${ }^{[c, d]}$ Yu Lei, ${ }^{[d]}$ Mauricio Terrones, ${ }^{*}[d]$ and Vivechana Agarwal ${ }^{*[a]}$

*Corresponding author email: vagarwal@uaem.mx

\section{Materials and methods}

The citric acid (ACS reagent, $\geq 99.5 \%$ ), Urea (ACS reagent, 99 to $100 \%$ ), $\mathrm{H}_{2} \mathrm{SO}_{4}$, sodium borohydride $\left(\mathrm{NaBH}_{4}, 99.99 \%\right), \mathrm{HAuCl}_{4} 3 \mathrm{H}_{2} \mathrm{O}$, 4-nitrophenol, and methylene blue (spectrophotometric grade) were acquired from Sigma Aldrich and used without further treatment. Deionized water $(18.2 \mathrm{M} \Omega \mathrm{cm})$ was used throughout the experiments.

\section{Synthesis of nitrogen-doped graphene oxide dots (NGODs)}

Like the previously reported synthesis method [1], carbon dots were fabricated using two different hydrothermal and microwave heat treatment techniques. Briefly, bright blue emissive NGODs were fabricated using $125 \mathrm{mg}$ citric acid (CA) and $185 \mathrm{mg}$ urea suspension 
in $25 \mathrm{~mL}$ deionized (DI) water. The reaction mixture was then stirred for $10 \mathrm{~min}$ to make a clear solution and then kept in a Teflon-lined hydrothermal autoclave at $180^{\circ} \mathrm{C}$ for one hour. The final product (bright yellow color solution) was centrifuged several times by continuous washing with water/ethanol and stored under ambient conditions for further use.

\section{Synthesis of nitrogen doped graphene quantum dots (NGQDs)}

Nitrogen doped graphene quantum dots were synthesized using CA and urea in DI with a CA's fixed weight ratio to urea and microwave power [2]. Typically, a 1:1 wt ratio of CA and urea were dissolved into $3 \mathrm{~mL}$ distilled water and stirred for 20 min to make a clear solution. The clear solution was then heated in a microwave at $280 \mathrm{~W}$ for the $40 \mathrm{~s}$ to form a dark yellow semi-solid precipitate (i.e., NGQDs). The formed product was kept in ambient conditions and then diluted with $2 \mathrm{~mL}$ of DI water under constant stirring for $0.5 \mathrm{~h}$. The obtained product was centrifuged several times by continuous washing with water/ethanol to remove excess/unreacted precursors, then NGQDs were stored at ambient conditions to further analysis.

\section{Synthesis of Gold nanoparticles (AuNPs)}

Modified sodium citrate reduction technique proposed by Turkevich was utilized to fabricate $\mathrm{Au}$ nanoparticles [3]. In brief, an aqueous gold ion $\left(\mathrm{HAuCl}_{4} \cdot 3 \mathrm{H}_{2} \mathrm{O}\right)$ solution was prepared by dissolving $9.86 \mathrm{mg}$ of gold ions in $25 \mathrm{~mL}$ DI water. The solution was heated up to its boiling temperature (around $95^{\circ} \mathrm{C}$ ) in a glass beaker. Once the solution starts boiling, the beaker was taken out of the heating mantle. A sodium citrate solution $(28.5 \mathrm{mg}$ dispersed into $2.5 \mathrm{~mL}$ of DI water) was slowly added to it under continuous magnetic agitation. Within $10 \mathrm{~min}$, the reaction mixer's visual color was transformed from pale yellow to violet, revealing the formation of AuNPs. Further, the synthesized AuNPs were obtained by centrifugation processes. The AuNPs powder sample was stored for further use. 


\section{Surface characterization}

The optical properties, including UV-visible and fluorescence spectra of as-synthesized NGODs, were measured in a dual-beam Perkin-Elmer Lambda 950 and a Cary Eclipse Fluorescence Spectrophotometer, respectively. Transmission electron microscopy (TEM) was carried out with a JEOL JEM-ARM200F transmission electron microscope to identify the morphology and particle size distribution of NGODs. The sample was prepared on lacey carbon film over 100 mesh $\mathrm{Cu}$ grid by drop-casting well diluted NGODs and evaporated under ambient conditions. X-ray diffraction (XRD) patterns of NGODs and NGQDs were performed with a Bruker D8 X-ray diffractometer with $\mathrm{Cu} K \alpha$ radiation $(\lambda=1.54 \AA)$. XRD samples were prepared as a film by drop-casting using $500 \mu \mathrm{L}$ of 4-nitrophenol with $5 \mu \mathrm{L}$ of as-prepared NGODs and then evaporated using a heating mantle at $45 \pm 5^{\circ} \mathrm{C}$. X-ray photoelectron spectroscopy (XPS) was recorded on an ESCA Ulvac-PHI 1600 photoelectron spectrometer from Physical Electronics using Al Ka radiation photon energy of $1486.6 \pm 0.2$ eV. The Fourier transform infrared (FTIR) spectra of NGODs and NGQDs were measured using a Varian 660-IR FT-IR spectrophotometer.

\section{Procedure for detection of gold nanoparticles (AuNPs) using NGODs}

To detect AuNPs, $810 \mu \mathrm{L}$ of reaction mixture contains different concentrations, from 4 to 40 $\mu \mathrm{g}$, of AuNPs and $10 \mu \mathrm{L}$ of as-prepared carbon dots. After constant stirring for $20 \mathrm{~s}$, the solution photoluminescence emission spectra were recorded using a fluorescence spectrophotometer $\left(\lambda_{\mathrm{ex}}=344 \mathrm{~nm}\right)$ under ambient conditions.

\section{Removal of 4-nitrophenol (4-NP) and methylene blue (MB) using AuNPs@NGODs}

In this experiment, AuNPs (40 $\mu \mathrm{g}) @$ NGODs (10:1 wt \%) were used as a hybrid catalyst and dispersed in $3 \mathrm{~mL}$ DI water mixed with $3 \mathrm{~mL}$ of aqueous $4-\mathrm{NP}(1 \mathrm{mM})$ to form a mixture solution. Subsequently, $0.6 \mathrm{~mL}$ of a freshly prepared $\mathrm{NaBH}_{4}$ solution $(0.1 \mathrm{M})$ was added to the earlier mixture. The 4-NP removal process was followed by monitoring the change in the solution's optical absorption spectra in the 314 to $400 \mathrm{~nm}$ spectral range. A quartz cuvette of $1 \mathrm{~cm} \times 1 \mathrm{~cm} \times 5 \mathrm{~cm}$ dimension was utilized for recording the absorption spectra of the test solutions, a similar procedure used for the removal of methylene blue (10 ppm). 

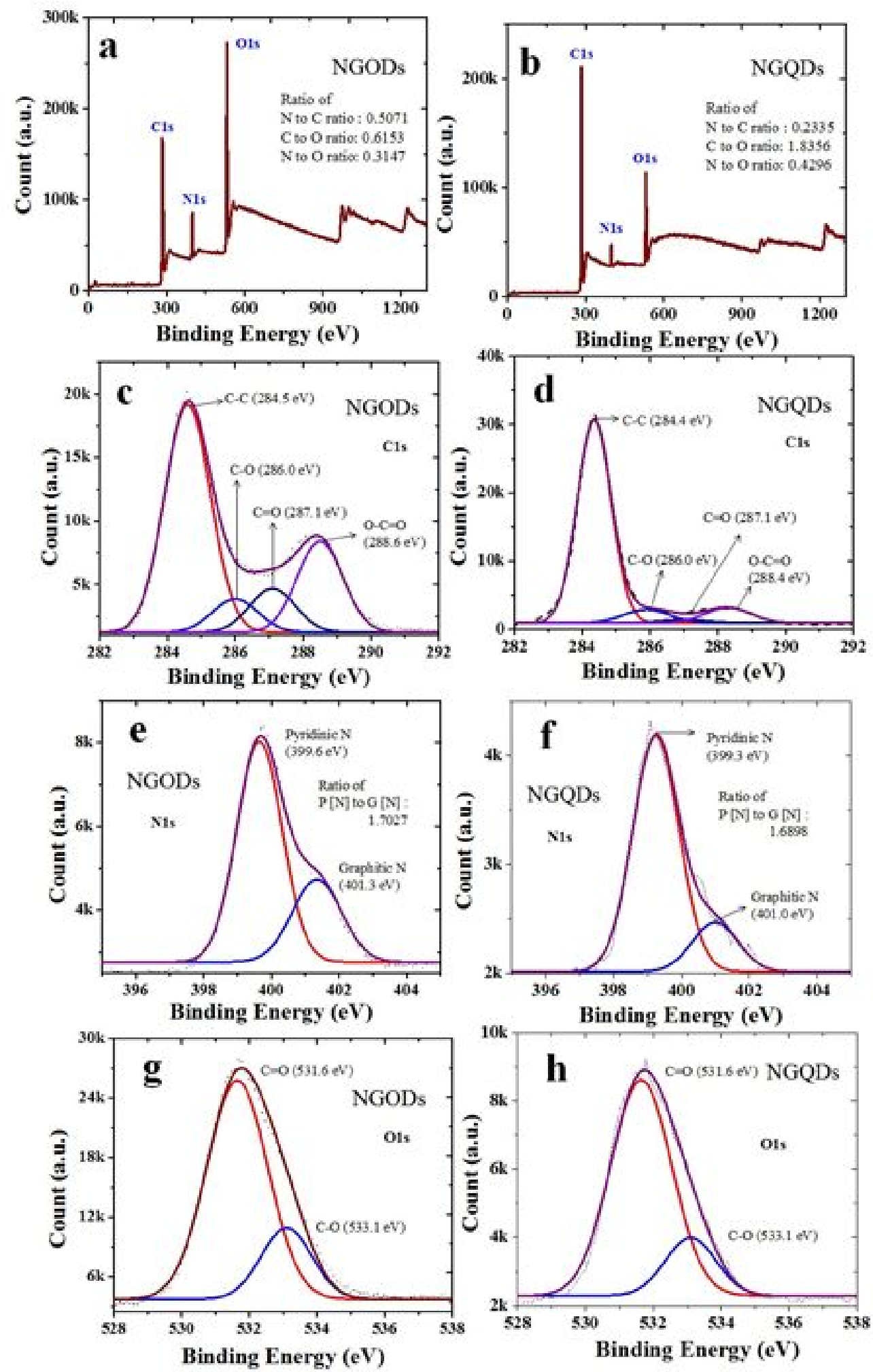

Figure S1. (a, b) XPS survey scan and deconvoluted XPS spectra of (c, d) C 1s, (e, f) N 1s and $(\mathrm{g}, \mathrm{h}) \mathrm{O} 1 \mathrm{~s}$ peaks of NGODs and NGQDs 


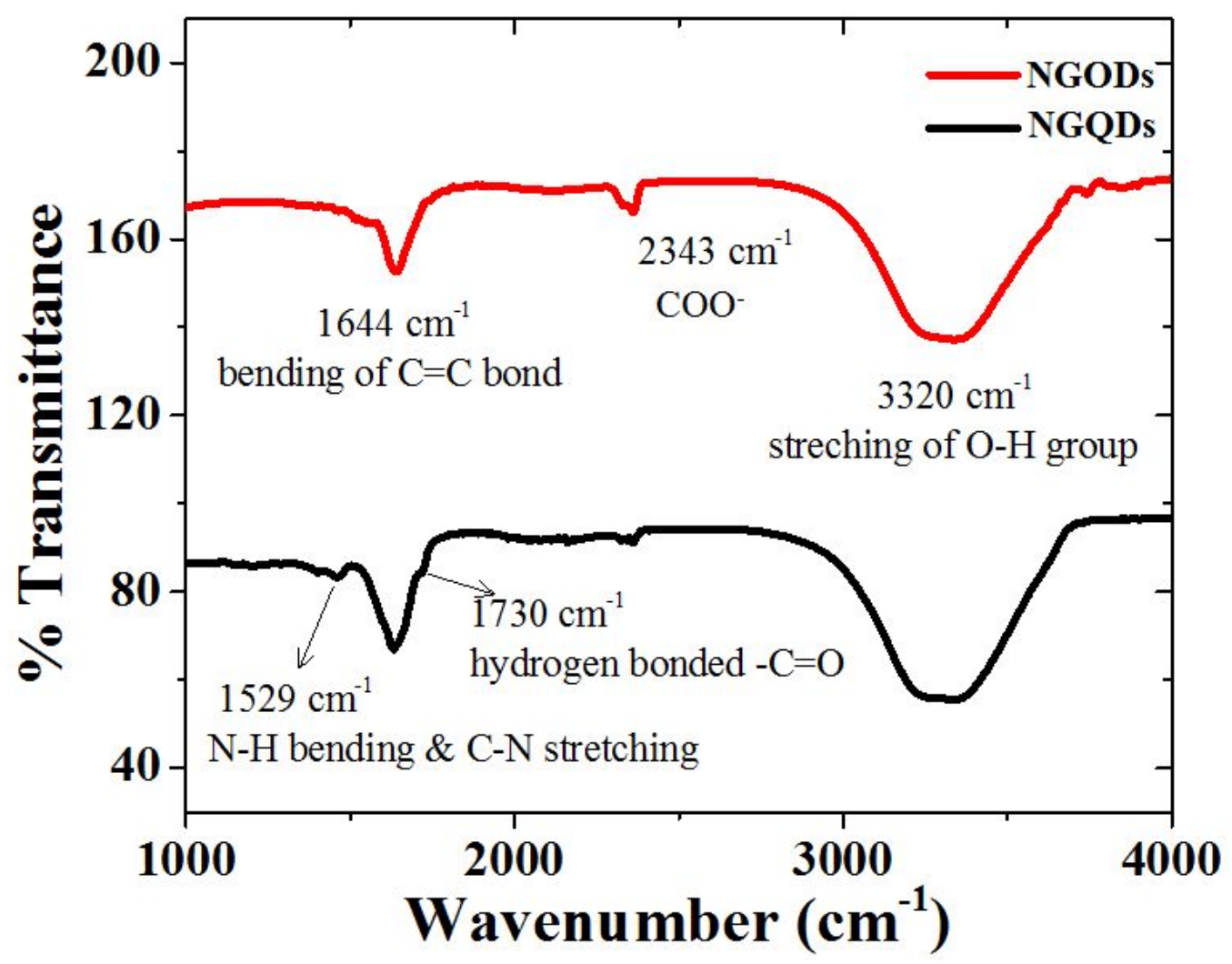

Figure S2. FTIR analysis of NGODs (red) and NGQDs (black) 


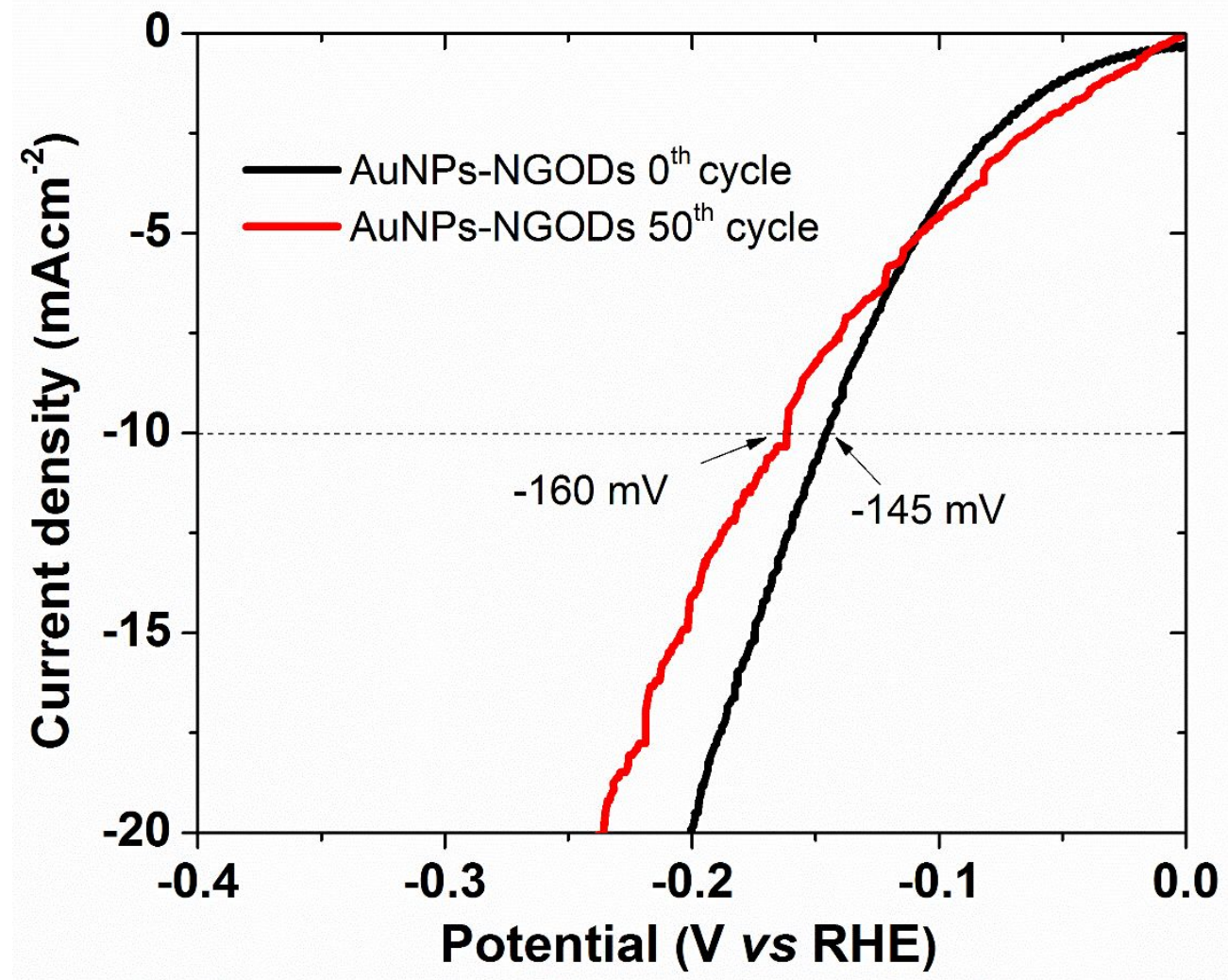

Figure S3. Electrocatalytic hydrogen evolution circulation performance: LSV polarization curves NGODs-AuNPs up to 50 cycles. 


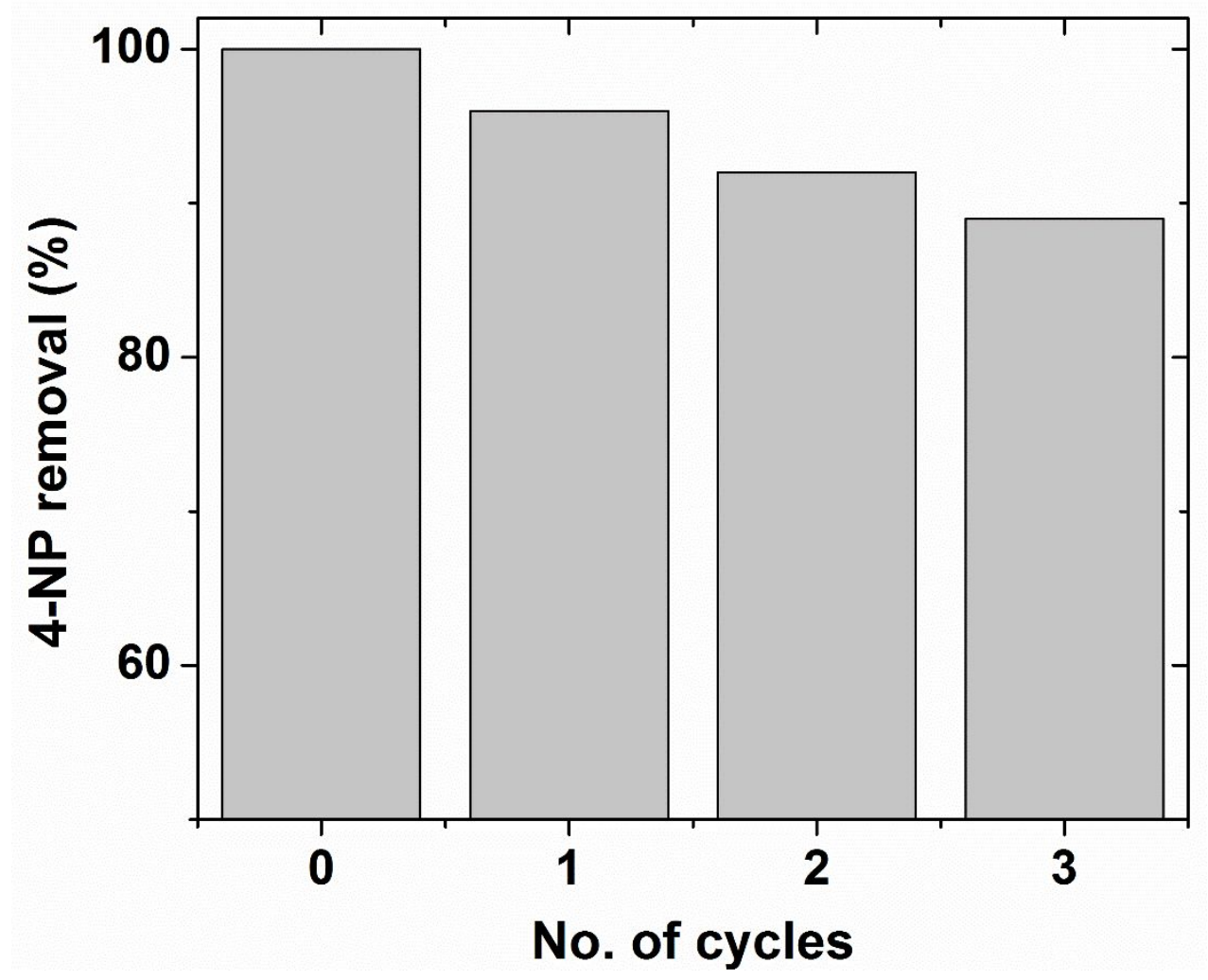

Figure S4. Reusability of the AuNPs-NGODs for the reduction of 4-nitrophenol up to 3 cycles. 


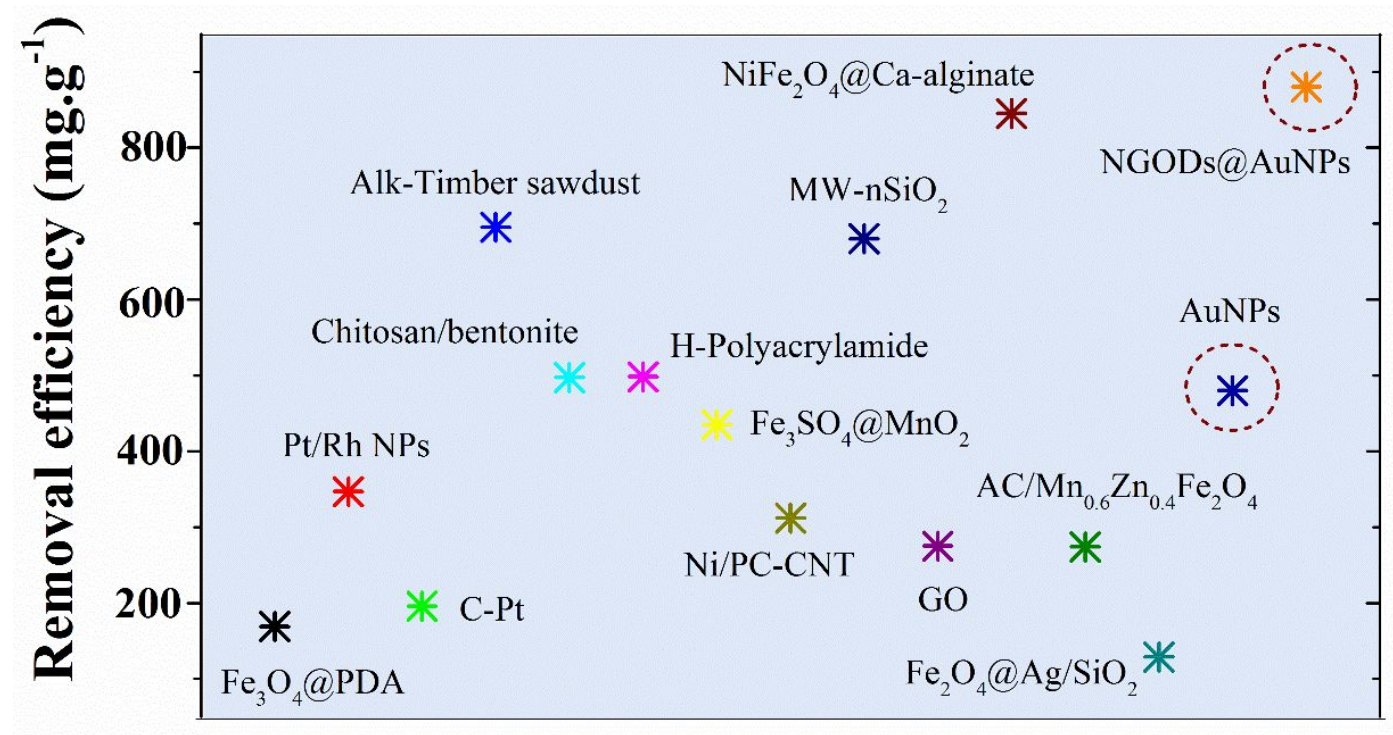

Figure S5. Degradation of methylene blue: comparison of removal efficiency $(\mathrm{mg} / \mathrm{g})$ with various metal-carbon based catalysts [4-16]. 


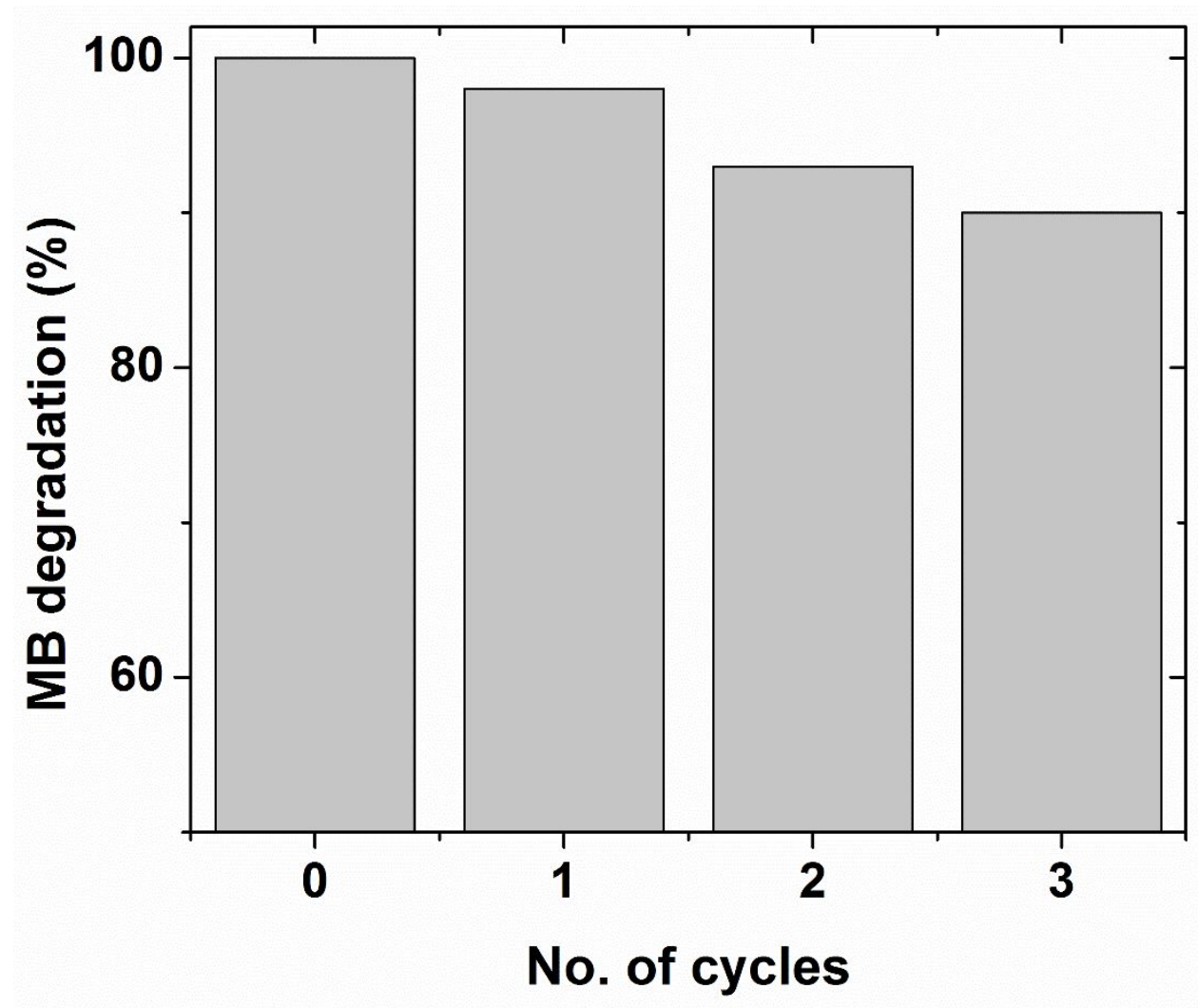

Figure S6. Reusability of the AuNPs-NGODs for the degradation of methylene blue up to 3 cycles. 


\section{Density functional theory details}

The calculations were performed out at the density functional theory (DFT) level. We used a first principle calculation method as implemented in Vienna Ab-initio Simulation Package (VASP) package $[4,5]$. This method is based on an augmented plane wave (PAW) using pseudopotentials [6]. The valence electronic configuration of $\mathrm{C}(4 \mathrm{~s} 3 \mathrm{~d}), \mathrm{O}(2 \mathrm{~s} 2 \mathrm{p}), \mathrm{N}(2 \mathrm{~s} 2 \mathrm{p})$, $\mathrm{Au}(5 \mathrm{~d} 6 \mathrm{~s})$, and $\mathrm{H}(1 \mathrm{~s})$ was expanded by using a set of plane waves with an energy cut-off of $520 \mathrm{eV}$. To avoid spurious interactions during the simulation, a van der Waals interaction with DFT-D2 Grimme parameterization was applied to correct the long-range interaction, which occurs during the propagation of such wave function materials [7]. For the systems under study, a self-consistent (SCF) convergence is reached when the energy difference attains a value smaller than 10-6 eV. In contrast, precision for the final forces at all geometrical optimizations must reach a value less than $0.01 \mathrm{eV} / \AA$. A supercell containing 2 x 2 x 1 primitive cells of graphene and graphene oxide with a vacuum spacing of $30 \AA$ is chosen to identify the Brillouin zone (BZ) integration. According to the Monkhorst-Pack mesh [8], a sampling of $4 \times 4 \times 1$ and $9 \times 9 \times 1$ was used to optimize geometries and the density of states calculation, respectively.

The efficiency of materials for high-performance hydrogen production was elucidated by using the notion of Gibbs free energy. This is a thermodynamic quantity that predicts the hydrogen evolution reaction (HER) in the materials under study. A suitable catalyst has a $\Delta G$ $=0 \mathrm{eV}$ (for instance: Pt is considered the best catalytic material for HER with a free energy of $0.06 \mathrm{eV})$. If $(\Delta G>0)$, indicates that the bond between hydrogen adsorbed on the surface is weak and the reaction is exothermic. Whereas if $(\Delta G<0)$, indicates that the reaction is endothermic and the bond is strong. In the present work, this thermodynamic parameter was determined from Eq. 1 [9]:

\section{$\Delta \boldsymbol{G}=\Delta \boldsymbol{E}_{\mathrm{ads} 2}+\Delta E_{\mathrm{ZPE}}-\mathrm{T} . \Delta \boldsymbol{S} \quad \mathbf{E q} \cdot \mathbf{1}$}

$\Delta E_{\text {ads2 }}$ is the adsorption energy of the hydrogen atom on different Au/substrates. The adsorption energy $E_{\text {ads } 1}$ is determined using the following equation: 
$\Delta \mathrm{E}_{\mathrm{ads} 1}=\mathrm{E}($ substrate $/ \mathrm{H})-\mathrm{E}($ substrate $)-1 / 2 \mathrm{E}\left(\mathrm{H}_{2}\right)$ is the energy of $\mathrm{H}$ adsorbed on the substrates, $\mathrm{E}$ (substrate) is the energy of the four support and $\mathrm{E}\left(\mathrm{H}_{2}\right)$ is the energy of $\mathrm{H}_{2}$ molecule in an isolated state.

Additionally, $\triangle \mathrm{E}_{\mathrm{ZPE}}$ is the zero-point energy. This value could be obtained by the vibrational frequency calculation of hydrogen atoms and isolated $\mathrm{H}_{2}$. The former corrects the free energy and provides accurate results. At hydrogen interaction, we found an approximate value of $0.02 \mathrm{eV}$. This value could be considered negligible. The change of the entropy of the system is given in Eq. 2 is T. $\Delta S$ [10]. For hydrogen atoms T. $\Delta \boldsymbol{S}=0.25 \mathrm{eV}$ at $\mathrm{T}=300 \mathrm{~K}$. This simple model could provide a high degree of accuracy to be compared with experimental results. We determined the adsorption energy of Au-NP on carbon substrates using the following equation:

\section{$E_{\text {ads2 }}=E($ substrate $+A u)-E($ substrate $)-E(A u) . E q .2$}

in which $\mathrm{E}($ substrate $+\mathrm{Au})$ is the energy of the Au-NP adsorbed on the substrate, E (substrate) is the energy of different substrates, and $\mathrm{E}(\mathrm{Au})$ is the energy of the Au NP in an isolated state. We performed the calculation for four systems; based on Au-NP adsorption on graphene, graphene oxide, N-doped graphene, and N-doped graphene oxide. The systems under study consist of a Au-NP adsorbed on the carbon systems. The number of atoms in the Au-NP is 7 , as shown in Fig. 7 (a).

The charge density difference and planar-averaged charge density difference were performed using the same DFT framework. The charge transfer analysis of the Au NP on substrates with and without $\mathrm{N}$-doping was analyzed using Bader population analysis [11, 12]. The isosurfaces of electronic density difference were mapped and determined with the following equation:

\section{$\Delta(\rho)=\rho($ substrate $+\mathbf{A u})-\rho($ substrate $)-\rho(\mathbf{A u}), \mathbf{E q} .3$}

In which, $\rho$ (substrate $+\mathrm{Au})$ is the charge of $\mathrm{Au} /$ substrate systems; $\rho$ (substrate) is the charge of the substrates and $\rho(\mathrm{Au})$ is the charge density of the isolated Au NP. The formula that 
gives the planar-averaged charge density difference along the z-direction, normal to the surface, is defined by:

$\Delta(\rho(\mathbf{z}))=\rho\{$ substrate $+\mathbf{A u}\}(\mathbf{z})-\rho\{$ substrate $\}(\mathbf{z})-\rho\{\mathbf{A u}\}(\mathbf{z}), \mathbf{E q} .4$

in which $\rho\{$ substrate $+\mathbf{A u}\}(\mathrm{z}), \rho\{$ substrate $\}(\mathrm{z}) \$$ and $\rho\{\mathrm{Au}\}(\mathrm{z})$, are the planar-averaged charge density of the $\mathrm{Au} /$ substrates, the support, and the isolated $\mathrm{Au} \mathrm{NP}$, respectively.

(a)

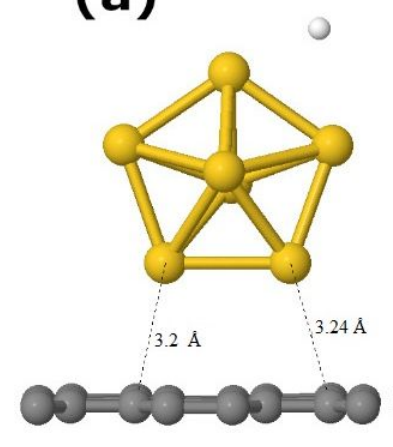

(c)

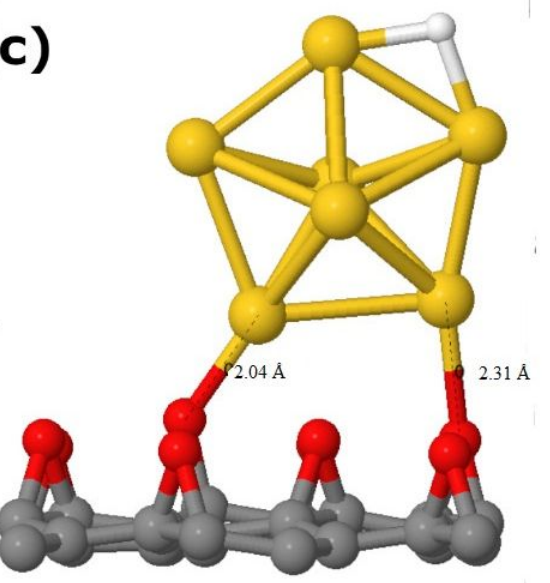

(b)
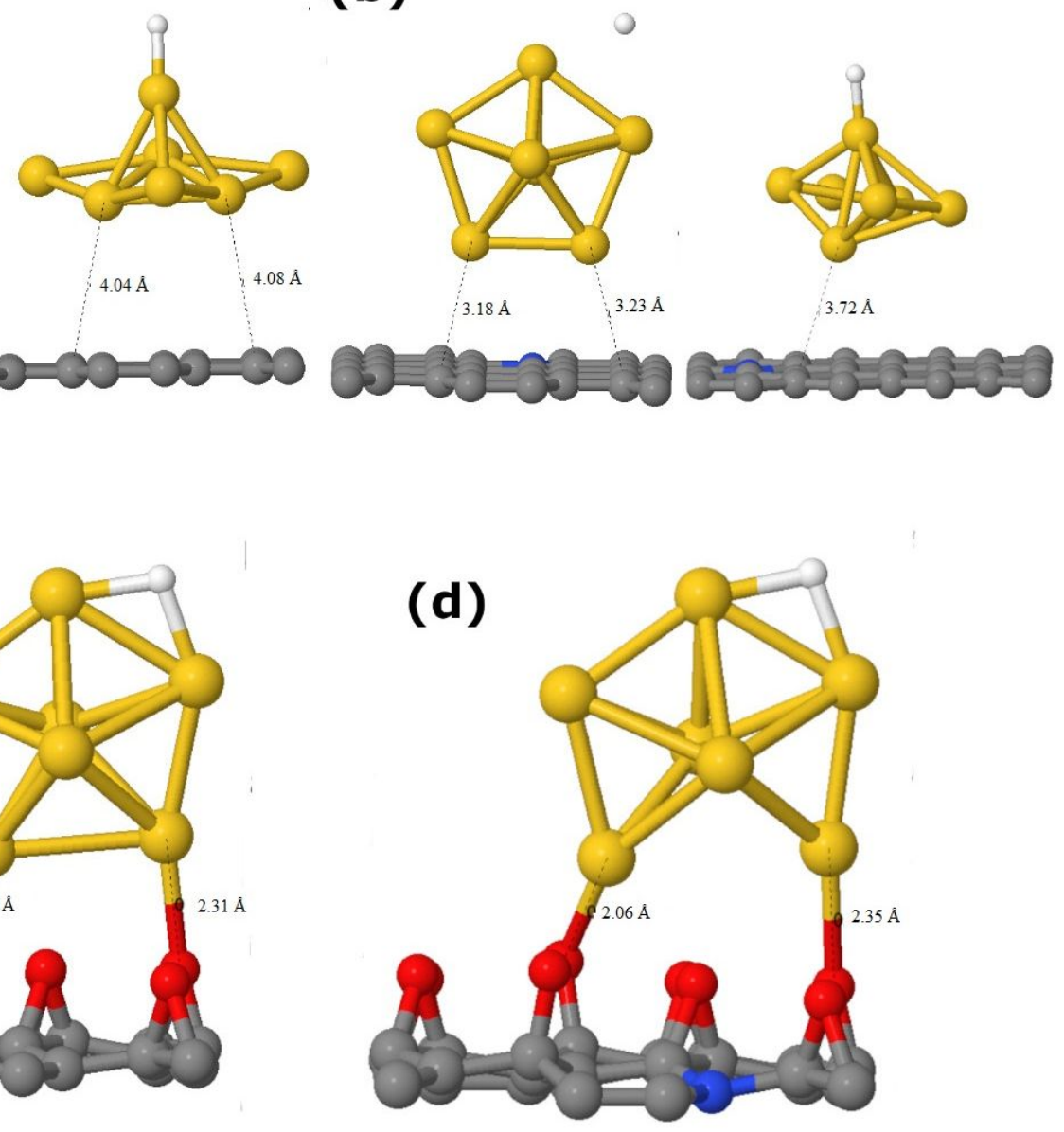

Figure S7: (a) The geometry structure of Au NP. The optimized geometry structures of $\mathrm{H}$ on (b) $\mathrm{Au} / \mathrm{GR}$ (c) $\mathrm{Au} / \mathrm{NGR}$, (d) Au/GRO, and (e) Au/NGRO. The Au, C, O, and $\mathrm{H}$ atoms are 
depicted in yellow, grey, red, and white balls. The calculations were perfomed using DFTPBE $+v D W$ approximation. In Fig. (c) and (d), The optimized structrues show no planar structure due to the presence of the oxygen functional groups
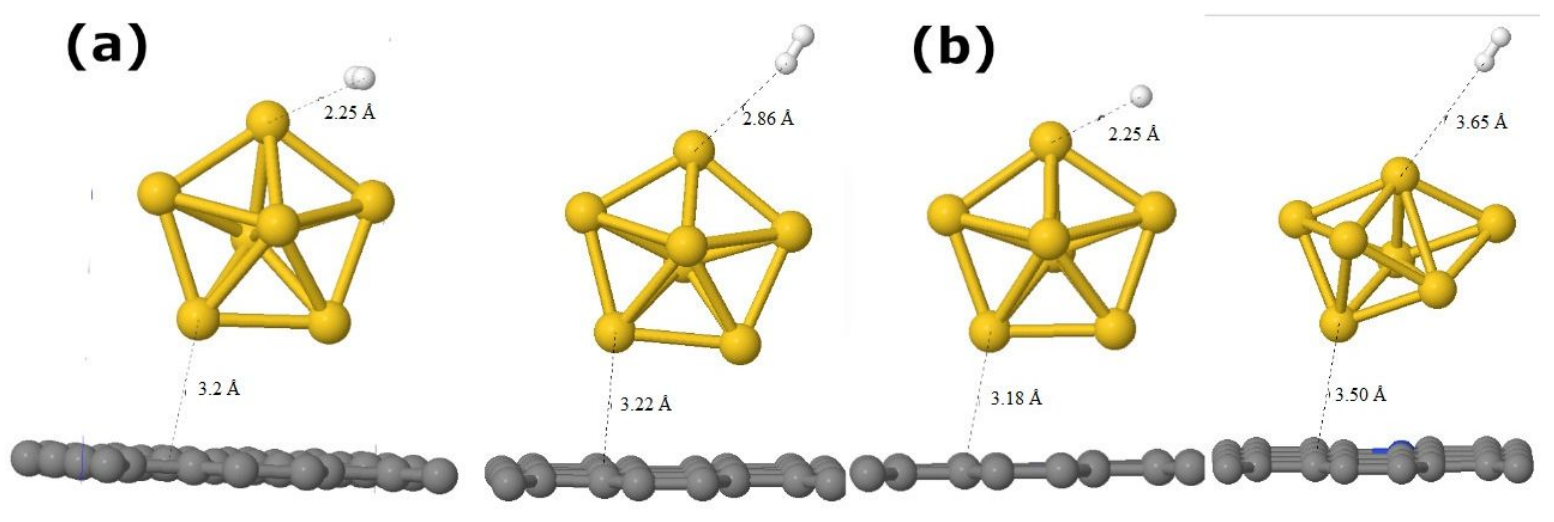

(c)
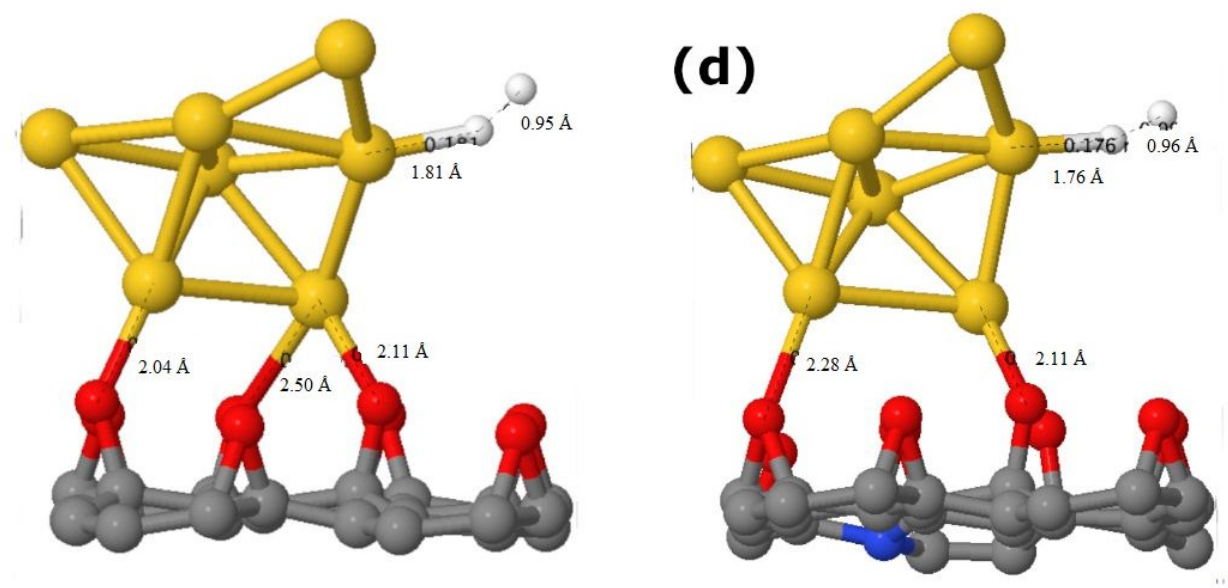

Figure S8: The optimized geometry structures of $\mathrm{H}_{2}$ on (a) $\mathrm{Au} / \mathrm{GR}$, (b) $\mathrm{Au} / \mathrm{NGR}$, (c) $\mathrm{Au} / \mathrm{GRO}$, and (d) $\mathrm{Au} / \mathrm{NGRO}$. The $\mathrm{Au}, \mathrm{C}, \mathrm{O}$, and $\mathrm{H}$ atoms are depicted in yellow, grey, red and white balls, respectively. The calculations were perfomed using DFT-PBE+vDW approximation. In Fig.(c) and (d), The optimized structrues show no planar structure due to the presence of the oxygen functional groups. 
(a)
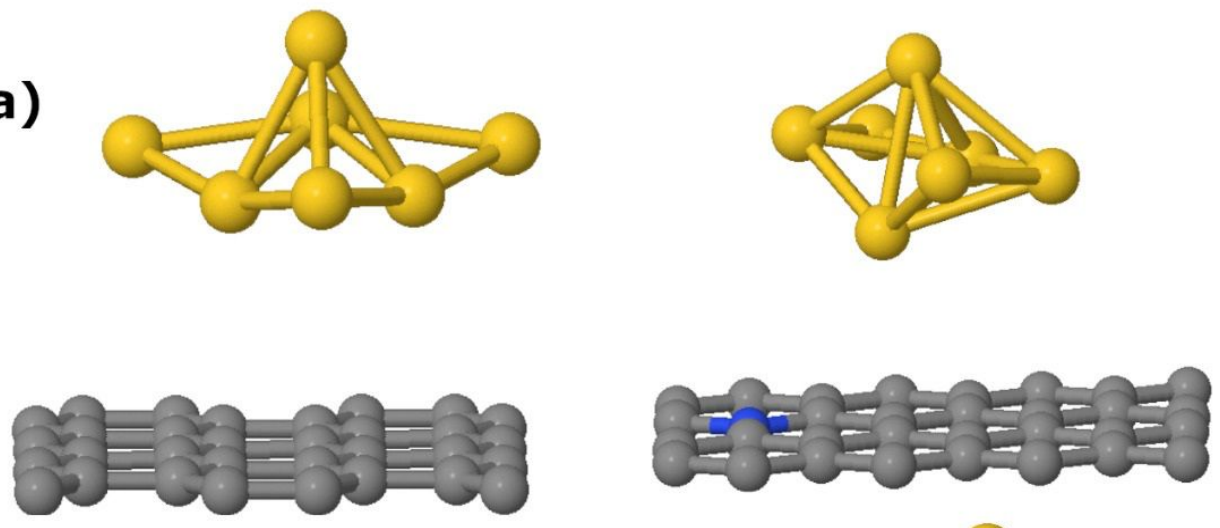

(b)
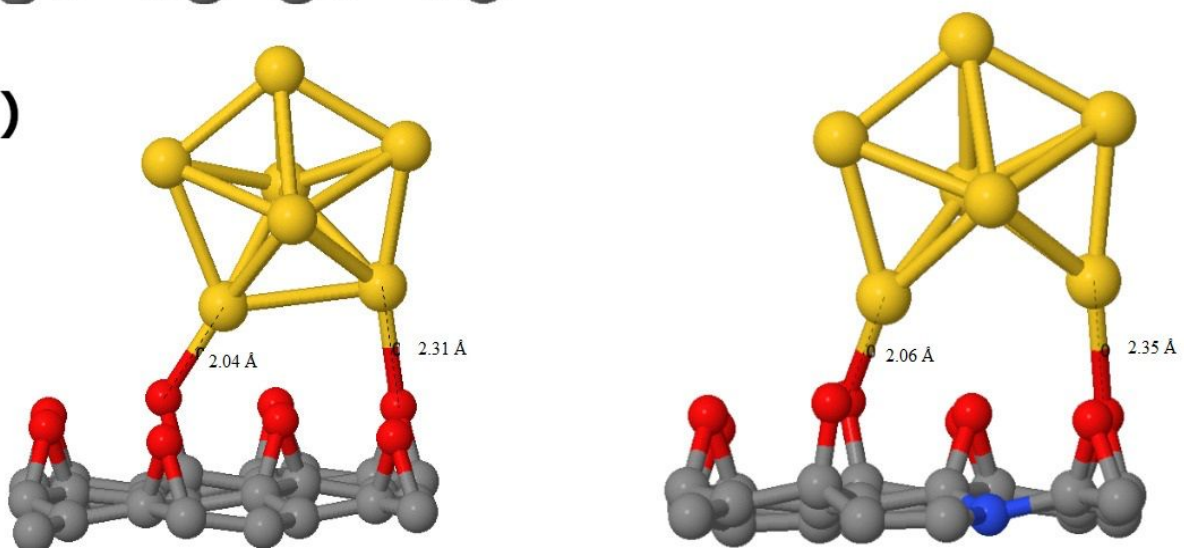

Figure S9: Optimized geometry structures on (a) GR and NGR (b) GRO and NGRO. The $\mathrm{Au}, \mathrm{C}, \mathrm{O}$, and $\mathrm{H}$ atoms are depicted in yellow, grey, red and white balls, respectively. The calculations were perfomed using DFT-PBE+vDW approximation. In Fig. (b), The optimized structrues show no planar structure due to the presence of the oxygen functional groups. 
(a) $\mathrm{Au} / \mathrm{G}$

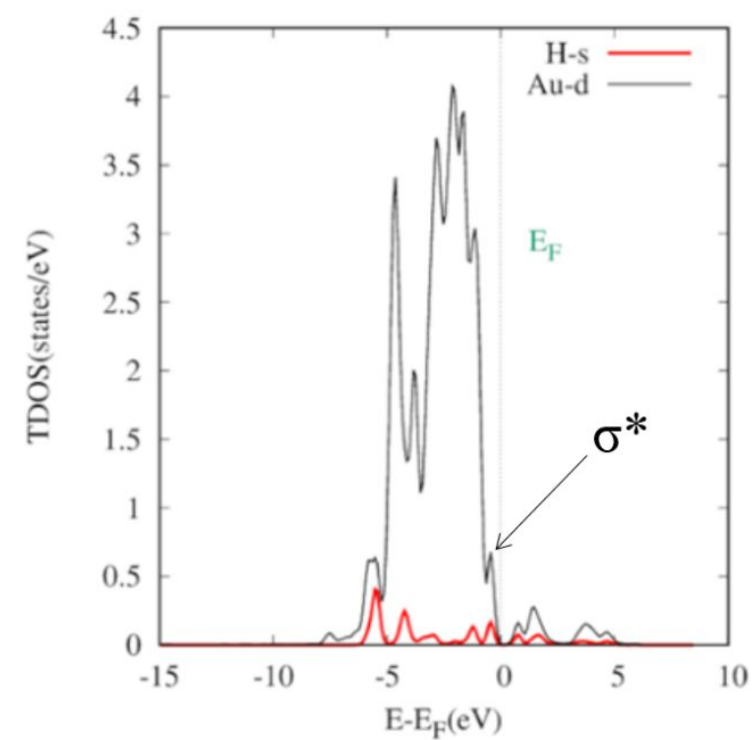

(c) $\mathrm{Au} / \mathrm{GO}$

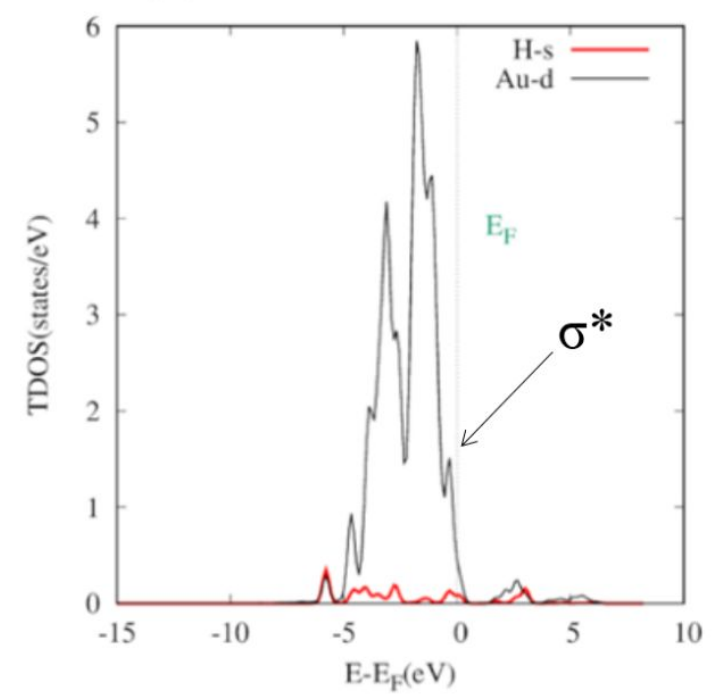

(b) $\mathrm{Au} / \mathrm{NG}$

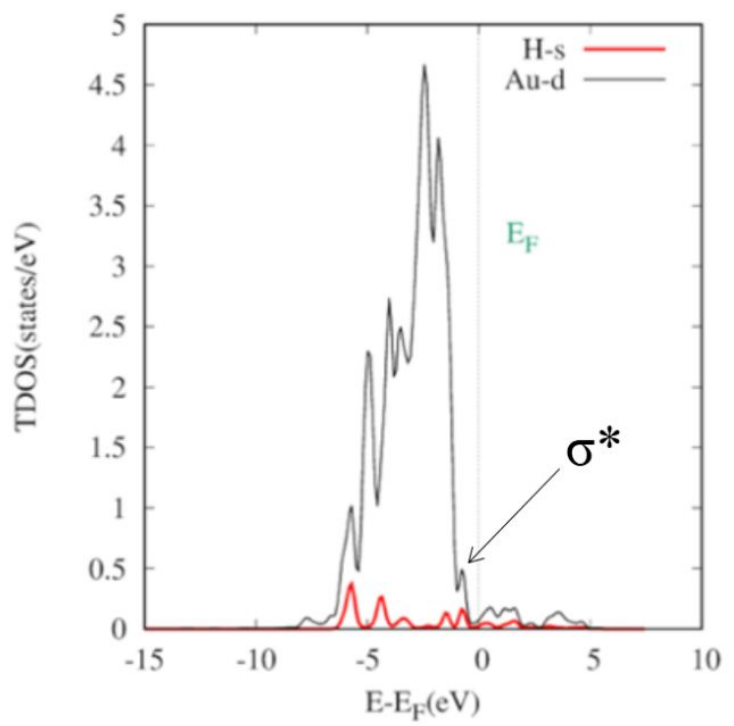

(d)Au/NGO

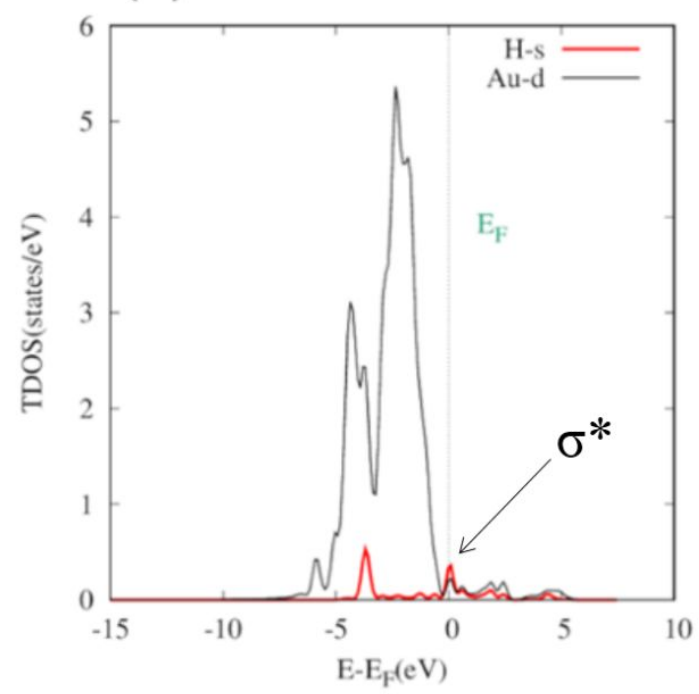

Figure S10: Projected density of states of $1 \mathrm{~s}-\mathrm{H}$ and 5d-Au for $\mathrm{H}$ on (a) Au/GR, (b) Au/NGR, (c) $\mathrm{Au} / \mathrm{GRO}$ and (d) Au/NGRO. The red and black lines represent the projected density of states of $\mathrm{H}-\mathrm{s}$ and $\mathrm{Au}-\mathrm{d}$, respectively.

In DOSs plots, we indiacted the psotion of the orbital sigma star, flech indictates different positions of the sigma star respect to the Fermi level, the Fermi level is indicated by a dahed vertical line and positioned at $0 \mathrm{eV}$. 
References:

1. Bogireddy, N.K.R., Cruz Silva, R., Valenzuela, M. A., Agarwal, V. Jour. Hazar. Mat. 2020, 386, 121643.

2. Bogireddy, N.K.R., Sotelo, R. S. E., Agarwal, V. Chem. Eng. Jour. 2021, 414, 128830.

3. Bogireddy, N. K. R., Umapada, P., Martinez, G. L., Agarwal, V. RSC Adv., 2018, 8, 24819-24826.

4. Wu, M. ; Li, Y. ; Yue, R.; Zhang, X.; Huang, Y. Sci. Rep. 2017, 7, 42773.

5. Yunus, Y.; Tugba, O. O.; Betu, I.; Bahdisen, G.; ultan, K.; Aysun, S., Enes, D.; Zeynep, D.; Hakan, S.; Fatih, S. Chem. Select 2017, 2 (2), 697-701.

6. Sert, H.; Yildiz, Y.; Okyay, T.; Sen, B.; Gezer, B.; Bozkurt, S.; Başskaya, G.; Sen, F. Journal of Nanoscience and Nanotechnology 2017, 17 (7), 4799-4804.

7. Yamina, D., El, H. E.; Abdallah, A.; Louis-Charles, M. Journal of Saudi Chemical Society 2016, 20 (1), S241-S249.

8. Dotto, G. L.; Rodrigues, F. K.; Tanabe, E. H.; Fröhlich, R.; Bertuol, D. A.; Martins, T. R.; Foletto, E. L. Jour. of Envi. Chem. Eng. 2016, 4 (3), 3230-3239.

9. Soumitra, G.; Asish, S.; Mohammad, R.; Asit, B. P.; Holger, S.; Sagar, P. ACS Appl. Mater. Interfaces 2014, 6 (7), 4766-4777.

10. Hadi, S.; Amir, A. A.; Rahbar, K. Jour. of Molecular Structure 2017, 1149, 199-205.

11. Lina, J.; Xiaoshuang, Z.; Xinye, Q.; Mingdong, D. Journal of Colloid and Interface Science 2018, 509, 245-253.

12. Enrique, C. P.; Jenifer, C. S.; Anaelise, M. C.; Ahmad, H. -B.; Guilherme, L. D. Journal of Environmental Chemical Engineering 2018, 6 (1), 649-659.

13. Chongyang, Q.; Lianqin, Z.; Yan, L.; Deyi, W. Journal of Colloid and Interface Science 2018, 517, 18-27. 
14. Corneliu, C.; Andra, C. H.; Petrisor, S.; Petronela, P.; Valeria, H. Reactive and Functional Polymers 2018, 125, 57-69.

15. Rui, W.; Jing, Y.; Qi, H. Chemical Engineering Research and Design 2018, 132, 215 225.

16. Jyoti, S.; Garg, V. K.; Gupta, R. K. Journal of Molecular Liquids 2018, 250, 413422.

17. Kresse, G., Furthmüller, J. Physical review B 1996, 54, 11169.

18. Kresse, G., Furthmüller, J. Computational materials science 1996, 6, 15-50.

19. Blöchl, P. E. Physical review B 1994, 50, 17953.

20. Grimme, S. J.Comp. Chem. 2006, 27, 1787-1799

21. Monkhorst, H. J., Pack, J. D. Physical review B 1976, 13, 5188.

22. Nørskov, J. K., Bligaard, T., Logadottir, A., Kitchin, J. R., Chen, J. G., Pandelov, S., Stimming, U. Journal of The Electrochemical Society 2005, 152, J23.

23. Sholl, D. S., Steckel, J. A., Wiley, 2009, 113 - 130.

24. Tang, W., Sanville, E., Henkelman, G. Journal of Physics: Condensed Matter 2009, 21, 084204.

25. Sanville, E., Kenny, S. D., Smith, R., Henkelman, G. Journal of computational chemistry, 2007, 28, 899-908. 